\title{
FAMILIES OF GROUP ACTIONS, GENERIC ISOTRIVIALITY, AND LINEARIZATION
}

\author{
HANSPETER KRAFT* \\ Mathematisches Institut \\ Universität Basel \\ Rheinsprung 21 \\ CH-4051 Basel, Switzerland \\ hanspeter.kraft@unibas.ch
}

\author{
PETER RUSSELL** \\ Department of \\ Mathematics and Statistics \\ McGill University \\ 805 Sherbrooke Street West \\ Montreal, QC, Canada, H3A 2K6 \\ russell@math.mcgill.ca
}

\begin{abstract}
Our base field is the field $\mathbb{C}$ of complex numbers. We study families of reductive group actions on $\mathbb{A}^{2}$ parametrized by curves and show that every faithful action of a non-finite reductive group on $\mathbb{A}^{3}$ is linearizable, i.e., $G$-isomorphic to a representation of $G$. The difficulties arise for non-connected groups $G$.

We prove a Generic Equivalence Theorem which says that two affine morphisms $p: S \rightarrow Y$ and $q: T \rightarrow Y$ of varieties with isomorphic (closed) fibers become isomorphic under a dominant étale base change $\varphi: U \rightarrow Y$. A special case is the following result. Call a morphism $\varphi: X \rightarrow Y$ a fibration with fiber $F$ if $\varphi$ is flat and all fibers are (reduced and) isomorphic to $F$. Then an affine fibration with fiber $F$ admits an étale dominant morphism $\mu: U \rightarrow Y$ such that the pull-back is a trivial fiber bundle: $U \times_{Y} X \simeq U \times F$.

As an application we give short proofs of the following two (known) results:

(a) Every affine $\mathbb{A}^{1}$-fibration over a normal variety is locally trivial in the Zariskitopology (see [KW85]).

(b) Every affine $\mathbb{A}^{2}$-fibration over a smooth curve is locally trivial in the Zariskitopology (see [KZ01]).
\end{abstract}

\section{Introduction and main results}

\section{Linearization}

Our base field is the field $\mathbb{C}$ of complex numbers. For a variety $X$ we denote by $\mathcal{O}(X)$ the algebra of regular functions on $X$, i.e., the global sections of the sheaf $\mathcal{O}_{X}$ of regular functions on $X$. An action of an algebraic group $G$ on $X$ is called linearizable if $X$ is $G$-equivariantly isomorphic to a linear representation of $G$. The "Linearization Problem" asks if every action of a reductive algebraic group $G$ on affine $n$-space $\mathbb{A}^{n}$ is linearizable. For $n=2$ the problem has a positive answer, due to the structure of the automorphism group of $\mathbb{A}^{2}$ as an amalgamated product. On the other hand, there exist non-linearizable actions on certain $\mathbb{A}^{n}$ for all non-

DOI: $10.1007 / \mathrm{s} 00031$

${ }^{*}$ Supported by SNF (Schweizerischer Nationalfonds).

** Supported by NSERC, Canada.

Received August 23, 2012. Accepted February 20, 2014.

Corresponding Author: H. Kraft, e-mail: hanspeter.kraft@unibas.ch. 


\section{HANSPETER KRAFT, PETER RUSSELL}

commutative connected reductive groups; see [Sch89], [Kno91]. The open cases are commutative reductive groups, in particular, tori, and commutative finite groups. For a survey on this problem we refer to the literature ([Kra96], [KS92]).

A very interesting case is dimension 3 , where no counterexamples have occurred so far. It is known that all actions of semisimple groups are linearizable ([KP85]) as well as $\mathbb{C}^{*}$-actions (see [KKMLR97]). The following result completes the picture of reductive group actions on $\mathbb{A}^{3}$.

Theorem A. Every faithful action of a non-finite reductive group on $\mathbb{A}^{3}$ is linearizable.

The remaining case of a finite group action on $\mathbb{A}^{3}$ seems to be a very difficult problem.

\section{Generic isotriviality}

One of the basic results of our paper is the following "generic isotriviality" of group actions (Theorem 2.2).

Theorem B. Let $\varphi: X \rightarrow Y$ be a dominant morphism where $X, Y$ are irreducible, and let $G$ be a reductive group acting on $X / Y$. Assume that the action of $G$ on the general fiber of $\varphi$ is linearizable. Then there is a dominant morphism of finite degree $\mu: U \rightarrow Y$ such that the fiber product $X \times_{Y} U$ is $G$ isomorphic to $W \times U$ over $U$ where $W$ is a linear representation of $G$ :

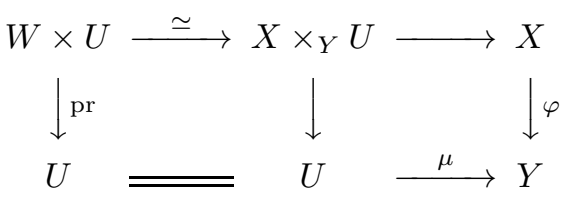

As usual, the condition that "the action of $G$ on the general fiber of $\varphi$ is linearizable" means that on an open dense subset of $Y$ all fibers $\varphi^{-1}(y)$ are reduced and $G$-isomorphic to a representation of $G$. Clearly, if the characteristic of the base field is zero, then the dominant map $\mu: U \rightarrow Y$ can be chosen to be étale.

Theorem B is based on a very general result, the "Generic Equivalence Theorem" which we formulate and prove in section 2. Several special cases of this result appear in the literature, quite often in connection with so-called "cylinder-like open sets", but the statement seems not to be known in this general form.

In the last paragraph we use this result to give a short and unified proof of the following results due to Kambayashi-Wright and Kaliman-Zaidenberg (Theorem 5.2).

\section{Theorem C.}

(a) If $\varphi: X \rightarrow Y$ is a flat affine morphism with fibers $\mathbb{A}^{1}$ and $Y$ normal, then $\varphi$ is a fiber bundle, locally trivial in the Zariski-topology.

(b) If $\varphi: X \rightarrow Y$ is a flat affine morphism with fibers $\mathbb{A}^{2}$ and $Y$ a smooth curve, then $\varphi$ is a fiber bundle, locally trivial in the Zariski-topology. 


\section{GENERIC ISOTRIVIALITY AND LINEARIZATION}

\section{Families of group actions}

An important concept and basic tool in our paper are families of automorphisms and families of group actions.

Definition 1.1. Let $Z, Y$ be varieties. A family of automorphisms of $Z$ parametrized by $Y$ is an automorphism $\Phi$ of $Z \times Y$ such that the projection pr: $Z \times$ $Y \rightarrow Y$ is invariant. We use the notation $\Phi=\left(\Phi_{y}\right)_{y \in Y}$ where $\Phi_{y}$ is the induced automorphism of the fiber $Z \times\{y\}$, which we identify with $Z$.

Similarly, for an algebraic group $G$, a family of $G$-actions on $Z$ parametrized by $Y$ is a $G$-action $\Phi$ on $Z \times Y$ such that the projection pr: $Z \times Y \rightarrow Y$ is $G$ invariant. Again we use the notation $\Phi=\left(\Phi_{y}\right)_{y \in Y}$ where $\Phi_{y}$ is the $G$-action on the fiber $Z \times\{y\}$ identified with $Z$.

Remark 1.1. It is known that the group $\operatorname{Aut}\left(\mathbb{A}^{n}\right)$ of polynomial automorphisms of affine $n$-space has the structure of an ind-group (see [FM10] or [Kum02]; this notion goes back to Shafarevich who called these objects infinite dimensional groups; see [Sha66], [Sha81], [Sha95]).

Using this structure it is easy to see that a family $\Phi=\left(\Phi_{y}\right)_{y \in Y}$ of automorphisms of $\mathbb{A}^{n}$ parametrized by $Y$ defines a morphism $\tilde{\Phi}: Y \rightarrow \operatorname{Aut}\left(\mathbb{A}^{n}\right), y \mapsto \Phi_{y}$, and vice versa. Similarly, a family of group actions of a reductive group $G$ parametrized by $Y$ is the same as a morphism $Y \rightarrow \operatorname{Mor}\left(G, \operatorname{Aut}\left(\mathbb{A}^{n}\right)\right)$ such that the image belongs to $\operatorname{Hom}\left(G, \operatorname{Aut}\left(\mathbb{A}^{n}\right)\right)$, where $\operatorname{Mor}\left(G, \operatorname{Aut}\left(\mathbb{A}^{n}\right)\right)$ also has a natural structure of an ind-variety.

We refer to the forthcoming paper [FK13] for a thorough investigation of automorphism groups of affine varieties.

Using an equivariant form of Sathaye's famous Theorem (see Lemma 3.3) we obtain the following result about linearization of families of two-dimensional representations (Theorem 3.2).

Theorem D. Let $G$ be a reductive group, and let $\Phi$ be a family of $G$-actions on $\mathbb{A}^{2}$ parametrized by a factorial affine curve $C$. Then the family is simultaneously linearizable, i.e., $\mathbb{A}^{2} \times C$ is $G$-isomorphic to $V \times C$ where $V$ is a two-dimensional linear representation of $G$.

This has the following consequence. Recall that a variable of $\mathbb{A}^{n}$ is a regular function $f$ on $\mathbb{A}^{n}$ which appears in an algebraically independent system of generators of the polynomial ring $\mathcal{O}\left(\mathbb{A}^{n}\right)$.

Corollary. A reductive group action on $\mathbb{A}^{3}$ fixing a variable is linearizable.

We conjecture that this holds in the more general situation where the reductive group action on $\mathbb{A}^{3}$ admits a semi-invariant variable.

\section{Generic equivalence and generic isotriviality}

Our first result concerns the generic equivalence of two morphisms having the same fibers. This holds under very general conditions. We formulated this result 


\section{HANSPETER KRAFT, PETER RUSSELL}

about 10 years ago, and it has circulated to some extent privately since. A preliminary write-up appeared in the Lecture Notes of an International School held in Hanoi in 2006; see [vdEVK07].

Generic Equivalence Theorem. Let $k$ be an algebraically closed field of infinite transcendence degree over the prime field. Let $p: S \rightarrow Y$ and $q: T \rightarrow Y$ be two affine morphisms where $S, T$ and $Y$ are $k$-varieties. Assume that for all $y \in Y$ the two (schematic) fibers $S_{y}:=p^{-1}(y)$ and $T_{y}:=q^{-1}(y)$ are isomorphic. Then there is a dominant morphism of finite degree $\varphi: U \rightarrow Y$ and an isomorphism $S \times{ }_{Y} U \simeq T \times{ }_{Y} U$ over $U$ :

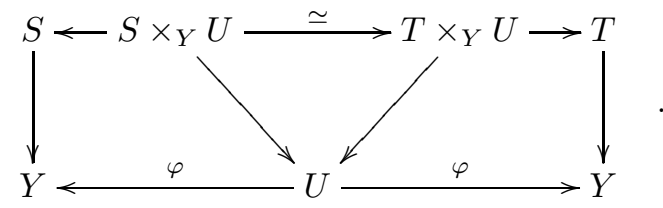

Remark 2.1. Under the assumptions of the theorem assume, in addition, that an algebraic group $G$ acts on $S$ and $T$ such that $p$ and $q$ are both invariant and that the isomorphisms $\varphi_{y}: S_{y} \stackrel{\sim}{\rightarrow} T_{y}$ can be chosen to be $G$-equivariant. Then the proposition holds $G$-equivariantly, i.e., there is a dominant morphism of finite degree $U \rightarrow Y$ and a $G$-equivariant isomorphism $S \times{ }_{Y} U \simeq T \times_{Y} U$.

Remark 2.2. We do not know if the Theorem holds for all algebraically closed fields, e.g., for $\overline{\mathbb{Q}}$.

The main ingredient for the proof is the following lemma which should be well known. Let $p: X \rightarrow Y$ be a dominant morphism between affine $k$-varieties where $k$ is algebraically closed and $Y$ irreducible. Then there is a field $k_{0} \subset k$ which is finitely generated over the prime field and a morphism $p_{0}: X_{0} \rightarrow Y_{0}$ of affine $k_{0}$-varieties with a cartesian diagram

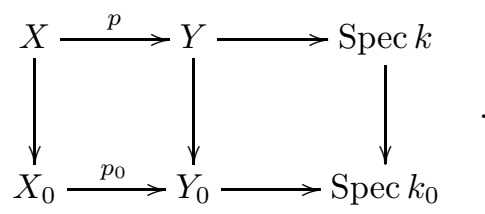

Lemma 2.1. In the notation above denote by $\omega$ : Spec $K_{0} \rightarrow Y_{0}$ the generic point of $Y_{0}$ and by $\left(X_{0}\right)_{\omega}:=p_{0}^{-1}(\omega)$ the generic fiber of $p_{0}$. Then every $k_{0}$-embedding $K_{0} \hookrightarrow k$ defines a closed point $y \in Y$ and an isomorphism

$$
\left(X_{0}\right)_{\omega} \times_{\operatorname{Spec} K_{0}} \operatorname{Spec} k \stackrel{\sim}{\rightarrow} X_{y}:=p^{-1}(y) .
$$

Proof. The $k_{0}$-embedding $\mathcal{O}\left(Y_{0}\right) \hookrightarrow K_{0} \hookrightarrow k$ defines a $k$-homomorphism $\mathcal{O}(Y)=$ $\mathcal{O}\left(Y_{0}\right) \otimes_{k_{0}} k \rightarrow k$, hence a closed point $\iota_{y}:\{y\} \rightarrow Y$, and we obtain the following commutative diagram where $X_{y}=p^{-1}(y)$ is the (schematic) fiber of $y$ : 


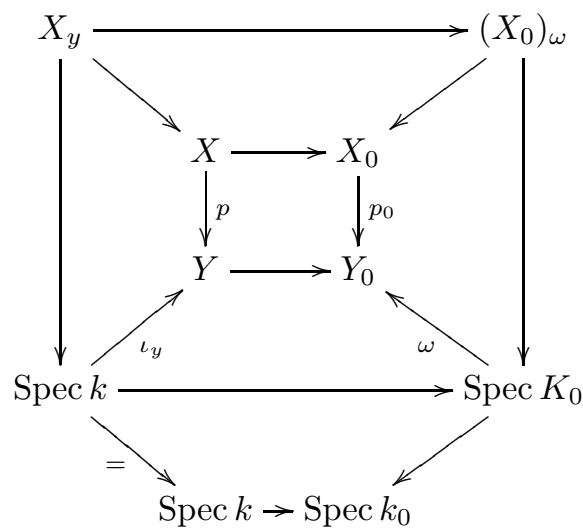

It follows that the outer diagram is cartesian:

$$
\left(X_{0}\right)_{\omega} \times_{\operatorname{Spec} K_{0}} \operatorname{Spec} k \simeq X_{0} \times_{Y_{0}} \operatorname{Spec} k \simeq X \times_{Y} \operatorname{Spec} k=X_{y}
$$

Proof of the Generic Equivalence Theorem. We can assume that $Y$ is affine and irreducible. Clearly, the whole setting is defined over a field $k_{0}$ which is finitely generated over the prime field. This means that there are $k_{0}$-varieties $Y_{0}, S_{0}, T_{0}$ and morphisms $p_{0}: S_{0} \rightarrow Y_{0}, q_{0}: T_{0} \rightarrow Y_{0}$ which become $p: S \rightarrow Y, q: T \rightarrow Y$ under the base change $k / k_{0}$, i.e., the following diagrams are cartesian:
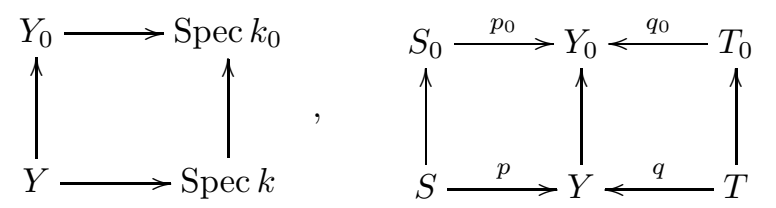

Let $\omega$ : Spec $K_{0} \rightarrow Y_{0}$ be the generic point of $Y_{0}$. By assumption on the field $k$ we can embed $K_{0}$ into $k$ (over $k_{0}$ ). According to Lemma 2.1 we get a closed point $\iota:\{y\} \rightarrow Y$ and isomorphisms

$$
\left(S_{0}\right)_{\omega} \times \text { Spec } K_{0} \operatorname{Spec} k \simeq S_{y} \simeq T_{y} \simeq\left(T_{0}\right)_{\omega} \times_{\operatorname{Spec} K_{0}} \operatorname{Spec} k
$$

This implies that there is a finite field extension $L_{0} / K_{0}$ and an isomorphism

$$
\left(S_{0}\right)_{\omega} \times_{\operatorname{Spec} K_{0}} \operatorname{Spec} L_{0} \simeq\left(T_{0}\right)_{\omega} \times_{\operatorname{Spec} K_{0}} \operatorname{Spec} L_{0} .
$$

In fact, in (1) we can first replace $k$ by a finitely generated $K_{0}$-algebra $A$ and then pass to $L_{0}:=A / \mathfrak{m}$ where $\mathfrak{m} \subset A$ is a maximal ideal contained in the image of the morphism $\left(S_{0}\right)_{\omega} \times$ Spec $K_{0} \operatorname{Spec} A \rightarrow \operatorname{Spec} A$.

What we proved here is the following well-known fact: Two affine algebraic $K_{0^{-}}$ schemes which are isomorphic over a field extension $k / K_{0}$ are already isomorphic over a finite field extension $L_{0} / K_{0}$. 


\section{HANSPETER KRAFT, PETER RUSSELL}

It follows that there is a finite field extension $L$ of $K=k(Y)$, the field of rational functions on $Y$, and an isomorphism

$$
S_{\omega} \times_{\operatorname{Spec} K} \operatorname{Spec} L \simeq T_{\omega} \times_{\operatorname{Spec} K} \operatorname{Spec} L
$$

where again $S_{\omega}$ and $T_{\omega}$ denote the generic fibers of $p$ and $q$ (over Spec $K$ ). Since $S_{\omega} \times \times_{\text {Spec } K} \operatorname{Spec} L=S \times_{Y} \operatorname{Spec} L$ there is a variety $X$ and a dominant morphism $X \rightarrow Y$ of finite degree $[L: K]$ such that $S \times_{Y} X \simeq T \times_{Y} X$.

Using the equivariant form of this result (see Remark 2.1 above) we can now prove Theorem B from the introduction.

Theorem 2.2. Let $\varphi: X \rightarrow Y$ be a dominant morphism where $X, Y$ are irreducible, and let $G$ be a reductive group acting on $X / Y$. Assume that the action of $G$ on the general fiber of $\varphi$ is linearizable. Then there is a dominant morphism of finite degree $\mu: U \rightarrow Y$ such that the fiber product $X \times_{Y} U$ is $G$ isomorphic to $W \times U$ over $U$ where $W$ is a linear representation of $G$ :

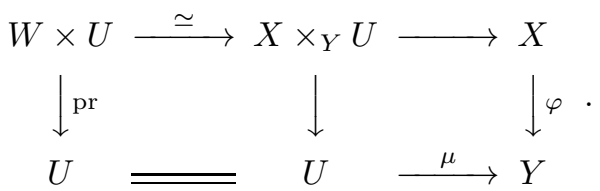

Proof. The assumptions of the theorem imply that there is an open dense set $U \subset X$ with the following properties:

(a) $U$ is smooth

(b) The fibers $\varphi^{-1}(u)$ for $u \in U$ are reduced and isomorphic to $\mathbb{C}^{n}$ where $n:=\operatorname{dim} Y-\operatorname{dim} X$.

(c) The action of $G$ on a fiber $\varphi^{-1}(u)$ for $u \in U$ is linearizable.

To finish the proof using the Equivariant Generic Equivalence Theorem (Remark 2.1) we have to show the following:

(d) For all $u \in U$ the fiber $\varphi^{-1}(u)$ is $G$-isomorphic to a fixed representation $W$ of $G$.

In fact, $\varphi: \varphi^{-1}(U) \rightarrow U$ is smooth and surjective and the tangent space $T_{x_{0}} Y$ in a fixed point $x_{0} \in \varphi^{-1}(U)^{G}$ has a $G$-stable decomposition $T_{x_{0}} Y=T_{x_{0}} F_{0} \oplus V$ where $F_{0}:=\varphi^{-1}\left(\varphi\left(x_{0}\right)\right)$, and $d \varphi_{x_{0}}: V \stackrel{\sim}{\rightarrow} T_{\varphi\left(x_{0}\right)} X$, since $G$ is reductive. Moreover, there is a $G$-equivariant morphism $\mu: T_{x} F_{0} \oplus V \rightarrow Y$ sending $(0,0)$ to $x_{0}$ which is étale in a neighborhood of $(0,0)$. This implies that for all fixed points $x$ in a neighborhood of $x_{0}$ the tangent representation $T_{x} F, F:=\varphi^{-1}(\varphi(x))$, is isomorphic to $T_{x_{0}} F_{0}$. Thus all fibers in a neighborhood of $\varphi\left(x_{0}\right)$ are $G$-isomorphic to the same representation.

\section{Families of group actions on $\mathbb{A}^{2}$}

We start with a crucial result on families of group actions on $\mathbb{A}^{2}$ where we use in an essential way the amalgamated product structure of $\operatorname{Aut}\left(\mathbb{A}^{2}\right)$. We do not know how to generalize this to higher dimension. 


\section{GENERIC ISOTRIVIALITY AND LINEARIZATION}

Lemma 3.1. Let $G$ be a reductive group and let $\Phi$ be a family of $G$-actions on $\mathbb{A}^{2}$ parametrized by $Y$ where $Y$ is an irreducible affine variety. Then there is an open dense set $U \subset Y$ such that the family $\left.\Phi\right|_{U}$ is equivalent to the constant family of a (2-dimensional) linear representation $V$ of $G$, i.e., there is a $G$-equivariant isomorphism of $\mathbb{A}^{2} \times U$ with $V \times U$.

Proof. It is known that $\operatorname{Aut}(K[x, y])$ has the structure of an amalgamated product for any field $K$ of characteristic zero ([vdK53]). This implies that every reductive $K$-group action on $\mathbb{A}_{K}^{2}$ is linearizable ([Kam79]). Taking for $K$ the field of fractions of $\mathcal{O}(Y)$ this means that there exist $x_{1}, y_{1} \in K[x, y]$ such that $K[x, y]=K\left[x_{1}, y_{1}\right]$ and that $K x_{1} \oplus K y_{1}$ is stable under $G_{K}$. Since every representation of $G_{K}$ is defined over $\mathbb{C} \subset K$ (see, e.g., [Jan03, Cor. II.2.9]) we can assume that $\mathbb{C} x_{1} \oplus \mathbb{C} y_{1}$ is $G$-stable. Clearly, there is a $t \in \mathcal{O}(Y)$ such that $\mathcal{O}(Y)_{t}[x, y]=\mathcal{O}(Y)_{t}\left[x_{1}, y_{1}\right]$ and so $\mathbb{A}^{2} \times U$ is isomorphic to $V \times U$ as $G$-varieties where $U:=Y_{t}$ and $V:=\left(\mathbb{C} x_{1} \oplus \mathbb{C} y_{1}\right)^{*}$, the dual representation.

Theorem 3.2. Let $G$ be a reductive group, and let $\Phi$ be a family of $G$-actions on $\mathbb{A}^{2}$ parametrized by a factorial affine curve $C$. Then the family is simultaneously linearizable, i.e., $\mathbb{A}^{2} \times C$ is $G$-isomorphic to $V \times C$ where $V$ is a two-dimensional linear representation of $G$.

Proof. Set $A:=\mathcal{O}(C)$ so that $\mathcal{O}\left(\mathbb{A}^{2} \times C\right)=A[x, y]=: R$. We have seen in Lemma 3.1 that there exist $t \in A$ and $x_{1}, y_{1} \in A_{t}:=A\left[t^{-1}\right]$ such that $A_{t}[x, y]=$ $A_{t}\left[x_{1}, y_{1}\right]$ and that $\mathbb{C} x_{1} \oplus \mathbb{C} y_{1}$ is $G$-stable. Clearly, $C$ is obtained from $C_{t}$ by adding a finite number of points: $C=C_{t} \cup\left\{c_{1}, c_{2}, \ldots, c_{k}\right\}$. Moreover, every open set $C_{j}:=C_{t} \cup\left\{c_{1}, c_{2}, \ldots, c_{j}\right\}$ is an affine factorial curve. Hence, by induction, we can assume that $C$ is obtained from $C_{t}$ by adding a single point $c_{0}$, i.e., that $t$ has a simple zero in $c_{0} \in C$ and that $A t \subset A$ is the maximal ideal at $c_{0}$. Now the claim follows from the next lemma. (We only need the special case where the $G$-action on $C$ is trivial.)

Lemma 3.3. Let $C$ be an affine smooth curve and let $G$ be a reductive group acting on $C$ and $X:=\mathbb{A}^{2} \times C$ such that the projection $p: X \rightarrow C$ is $G$-equivariant. Let $c_{0} \in C$ be a fixed point of $G$ and set $C^{\prime}:=C \backslash\left\{c_{0}\right\}$. Assume that the following holds:

(a) There is a generator $t$ of the maximal ideal $\mathfrak{m}_{c_{0}} \subset \mathcal{O}(C)$ such that $\mathbb{C} t$ is G-stable.

(b) $X^{\prime}:=p^{-1}\left(C^{\prime}\right)$ is $G$-isomorphic to the product $W \times C^{\prime}$ where $W$ is a twodimensional representation of $G$.

Then $X$ is $G$-isomorphic to $W \times C$.

Proof. Set $A:=\mathcal{O}(C)$ so that $\mathcal{O}\left(\mathbb{A}^{2} \times C\right)=A[x, y]=: R$. By assumption there exist $x_{1}, y_{1} \in R$ such that $R_{t}=A_{t}[x, y]=A_{t}\left[x_{1}, y_{1}\right]$ and that $\mathbb{C} x_{1} \oplus \mathbb{C} y_{1}$ is $G$ stable. Denoting by $\bar{x}_{1}, \bar{y}_{1}$ the residue classes in $\bar{R}:=R / R t=\mathbb{C}[x, y]$ we obtain a linear $G$-homomorphism $\rho: \mathbb{C} x_{1} \oplus \mathbb{C} y_{1} \rightarrow \mathbb{C} \bar{x}_{1}+\mathbb{C} \bar{y}_{1} \subset \bar{R}$. Dividing $x_{1}$ and $y_{1}$ by the same power of $t$ we can assume that $\rho$ is non-zero. If the image $\mathbb{C} \bar{x}_{1}+\mathbb{C} \bar{y}_{1}$ has dimension 1 then the kernel of $\rho$ is a one-dimensional representation $\mathbb{C} h$ of $G$, so that $\mathbb{C} x_{1} \oplus \mathbb{C} y_{1}=\mathbb{C} h \oplus \mathbb{C} h^{\prime}$ where $\mathbb{C} h^{\prime}$ is $G$-stable. Now we can divide $h$ by a 


\section{HANSPETER KRAFT, PETER RUSSELL}

suitable power of $t$. In this way we arrive at a situation where $\operatorname{dim}\left(\mathbb{C} \bar{x}_{1}+\mathbb{C} \bar{y}_{1}\right)=2$. Define

$$
\begin{aligned}
n\left(x_{1}, y_{1}\right) & :=\min \left\{n \geq 0 \mid t^{n} x \in A\left[x_{1}, y_{1}\right]\right\} \\
m\left(x_{1}, y_{1}\right) & :=\min \left\{m \geq 0 \mid t^{m} y \in A\left[x_{1}, y_{1}\right]\right\}
\end{aligned}
$$

and set

$$
N\left(x_{1}, y_{1}\right):=n\left(x_{1}, y_{1}\right)+m\left(x_{1}, y_{1}\right) .
$$

Then we find the following expressions

$$
t^{n\left(x_{1}, y_{1}\right)} x=\sum_{i, j} a_{i j} x_{1}^{i} y_{1}^{j}, \quad t^{m\left(x_{1}, y_{1}\right)} y=\sum_{i, j} b_{i j} x_{1}^{i} y_{1}^{j}
$$

where $a_{i j}, b_{i j} \in A$, and not all $a_{i j}$ and not all $b_{i j}$ belong to the maximal ideal At. If $N\left(x_{1}, y_{1}\right)=0$ we are done. Otherwise it follows from (2) that $\bar{x}_{1}, \bar{y}_{1}$ are algebraically dependent.

Denote by $F \in \mathbb{C}[w, z]$ the minimal equation $F\left(\bar{x}_{1}, \bar{y}_{1}\right)=0$. Clearly, $F$ is the generator of the kernel of the canonical homomorphism $\varphi: \mathbb{C}[w, z] \rightarrow \bar{R}$ given by $\varphi(w):=\bar{x}_{1}$ and $\varphi(z):=\bar{y}_{1}$. Now it follows from [Sat83, Rem. 2.1] that $\mathbb{C}\left[\bar{x}_{1}, \bar{y}_{1}\right] \subset$ $\bar{R}$ is a polynomial ring in one variable, or, equivalently, that $F$ is a variable in $\mathbb{C}[w, z]$, i.e., there is an $H \in \mathbb{C}[w, z]$ such that $\mathbb{C}[w, z]=\mathbb{C}[F, H]$.

If we define a (linear) $G$-action on $\mathbb{C}[w, z]$ by using the same matrices as for the representation on $\mathbb{C} x_{1} \oplus \mathbb{C} y_{1}$, then the homomorphism $\varphi$ is obviously $G$-equivariant, hence the kernel is $G$-stable. This implies that $\mathbb{C} F \subset \mathbb{C}[w, z]$ is $G$-stable. Now we use the fact that there is a uniquely defined second variable $H \in \mathbb{C}[w, z]$ (up to an additive constant) which has lower degree than $F$ (see [Sat83, Thm. 3(1)]). It follows that $\mathbb{C} H+\mathbb{C} \subset \mathbb{C}[w, z]$ is $G$-stable and so $\mathbb{C}(H+\alpha)$ is $G$-stable for a suitable $\alpha \in \mathbb{C}$.

Putting $x_{2}:=F\left(x_{1}, y_{1}\right) \in R$ and $y_{2}:=H\left(x_{1}, y_{1}\right)+\alpha \in R$ we see that $\mathbb{C} x_{2}$ and $\mathbb{C} y_{2}$ are $G$-stable lines in $A$. Moreover, we have $\mathbb{C}\left[x_{2}, y_{2}\right]=\mathbb{C}\left[x_{1}, y_{1}\right] \subset R$ and so $A\left[x_{2}, y_{2}\right]=A\left[x_{1}, y_{1}\right]$. Since $\bar{x}_{2}=F\left(\bar{x}_{1}, \bar{y}_{1}\right)=0$ we can divide $x_{2}$ by a suitable power of $t$ such that $x_{3}:=x_{2} / t^{s} \in R \backslash R t$ for some $s>0$. Similarly, $y_{3}:=y_{2} / t^{r} \in R \backslash R t$ for some $r \geq 0$.

In order to see that this procedure will finally stop we calculate the number $N\left(x_{3}, y_{3}\right)$. Since $A\left[x_{2}, y_{2}\right]=A\left[x_{1}, y_{1}\right]$ we have $n\left(x_{2}, y_{2}\right)=n\left(x_{1}, y_{1}\right)$ and $m\left(x_{2}, y_{2}\right)=m\left(x_{1}, y_{1}\right)$, and one of them is $>0$, say $n\left(x_{1}, y_{1}\right)>0$. Using the first equation in (2) for $x_{2}=t^{s} x_{3}$ and $y_{2}=t^{r} y_{3}$ we see that $\overline{\sum_{j} a_{0 j} y_{2}^{j}}=0$. It follows that either $r>0$ or $\overline{a_{0 j}}=0$ for all $j$. In both cases we can divide both sides of the equation by $t$ and so $n\left(x_{3}, y_{3}\right)<n\left(x_{2}, y_{2}\right)$, hence $N\left(x_{3}, y_{3}\right)<N\left(x_{1}, y_{1}\right)$.

Remark 3.1. The crucial step in the proof above is Sathaye's result showing that $\mathbb{C}\left[\bar{x}_{1}, \bar{y}_{1}\right] \subset \mathbb{C}[x, y]$ is a polynomial ring in one variable in case $\bar{x}_{1}, \bar{y}_{1}$ are algebraically dependent. It is interesting to remark that this result is not needed in case $G$ is non-commutative, since there is no faithful action of a non-commutative group $G$ on $\mathbb{C}\left[\bar{x}_{1}, \bar{y}_{1}\right]$ in case this algebra is of dimension 1 , because there is no faithful action of $G$ on a rational curve (rational since $\mathbb{C}\left[\bar{x}_{1}, \bar{y}_{1}\right] \subset \mathbb{C}[x, y]$ ). 


\section{GENERIC ISOTRIVIALITY AND LINEARIZATION}

\section{Linearization of group actions on $\mathbb{A}^{3}$}

We now give the proof of Theorem A from the introduction stating that every faithful action of a non-finite reductive group $G$ on $\mathbb{A}^{3}$ is linearizable. The difficult part is the case of a non-connected group $G$ with $G^{0} \simeq \mathbb{C}^{*}$.

Proof of Theorem A. (a) If $G^{0}$ is not isomorphic to $\mathbb{C}^{*}$, then $G^{0}$ either contains $\mathbb{C}^{*} \times \mathbb{C}^{*}$ or a simple group of type $A_{1}$. It is well known that a faithful action of a $d$-dimensional torus has $d$-dimensional orbits, and that a non-trivial action of $\mathrm{SL}_{2}(\mathbb{C})$ on an affine variety has orbits of dimension $\geq 2$. It follows that $G$ admits orbits of dimension $\geq 2$ in $\mathbb{A}^{3}$, hence $\operatorname{dim} \mathbb{A}^{3} / / G \leq 1$. Now linearization follows from the results in [KS92]. In fact, Luna's Slice Theorem implies that any action of a reductive group on $\mathbb{A}^{n}$ with a zero-dimensional quotient $\mathbb{A}^{n} / / G$ is linearizable (cf. [KP85, 5.1 Prop.]). If $\operatorname{dim} \mathbb{A}^{n} / / G=1$ and if the action is fix-pointed, i.e., $\operatorname{dim}\left(\mathbb{A}^{n}\right)^{G}=1$, then linearization also follows (see [KS92, Chap. II.0.3, Cor.]); this goes back to $[\mathrm{BH} 85]$. Finally, if $\operatorname{dim} \mathbb{A}^{3} / / G=1$ and $\operatorname{dim}\left(\mathbb{A}^{3}\right)^{G}=0$, then the result is stated in [KS92, Chap. I.2.4, Thm. 5(4)].

(b) If $G \simeq \mathbb{C}^{*}$, then this is the main result of [KKMLR97]. So we are left with the case of a non-connected $G$ such that $G^{0} \simeq \mathbb{C}^{*}$.

(c) We fix an identification $G^{0}=\mathbb{C}^{*}$. By (b) we can assume that the action of $\mathbb{C}^{*}$ is linear with weights $n_{1} \geq n_{2} \geq 0>n_{3}$, i.e., $t(x, y, z)=\left(t^{n_{1}} \cdot x, t^{n_{2}} \cdot y, t^{n_{3}} \cdot z\right)$, since in all other cases the quotient $\mathbb{A}^{3} / / G$ has dimension $\leq 1$, and so we are done by (a).

(d) Let us first consider the case where $n_{2}>0$. Then the hyperplane $U$ given by $z=0$ has the following description:

$$
U=\left\{v \in \mathbb{A}^{3} \mid \lim _{t \rightarrow 0} t v=0\right\}
$$

This implies that every $g \in G$ commutes with $\mathbb{C}^{*}$ and therefore stabilizes $U$. In fact, if $g$ does not commute with $\mathbb{C}^{*}$ then $g t g^{-1}=t^{-1}$ for all $t \in \mathbb{C}^{*}$ and so

$$
g U=\left\{v \in \mathbb{A}^{3} \mid \lim _{t \rightarrow \infty} t v=0\right\} .
$$

This is a contradiction since the right-hand side equals the line $\{x=y=0\}$. It follows that $\mathbb{C} z \subset \mathcal{O}\left(\mathbb{A}^{3}\right)$ is $G$-stable: $g z=\chi(g) \cdot z$ where $\chi$ is a character of $G$. Thus the projection $p: \mathbb{A}^{3} \rightarrow \mathbb{C}_{\chi},(x, y, z) \mapsto z$, is $G$-equivariant. Define $H:=\operatorname{ker} \chi$ and set $\dot{\mathbb{C}}:=\mathbb{C}_{\chi} \backslash\{0\}$. Then $p^{-1}(\dot{\mathbb{C}})$ is $G$-isomorphic to the associated bundle $B:=G *_{H} p^{-1}(1)$. The action of $H$ on $p^{-1}(1) \simeq \mathbb{A}^{2}$ is linearizable and so $B \simeq W \times \dot{\mathbb{C}}$ as an $H$-variety where $W$ is a two-dimensional representation of $H$ and $H$ acts trivially on $\dot{\mathbb{C}}$. Thus, by Lemma 3.3 , the action of $H$ on $\mathbb{A}^{3}$ is linearizable: $\mathbb{A}^{3}$ is $H$ isomorphic to $W \times \mathbb{C}$. In particular, the hyperplane $U$ is $H$ isomorphic to $W$ which implies that the representation of $H$ on $W$ can be extended to a representation of $G$. As a consequence, the associated bundle $B$ splits into a product:

$$
p^{-1}(\dot{\mathbb{C}}) \stackrel{\sim}{\longrightarrow} W \times \dot{\mathbb{C}}_{\chi} \text { as a } G \text {-variety. }
$$

Now we can again apply Lemma 3.3 and the claim follows. 


\section{HANSPETER KRAFT, PETER RUSSELL}

(e) We are left with the case $n_{2}=0$. Here we have the following two hyperplanes

$$
\begin{aligned}
U_{0} & :=\{z=0\}=\left\{v \in \mathbb{A}^{3} \mid \lim _{t \rightarrow 0} t v \text { exists }\right\}, \\
U_{\infty} & :=\{x=0\}=\left\{v \in \mathbb{A}^{3} \mid \lim _{t \rightarrow \infty} t v \text { exists }\right\} .
\end{aligned}
$$

Clearly, $U_{0} \cup U_{\infty}$ is stable under $G$ and therefore $\mathbb{C} x \oplus \mathbb{C} z \subset \mathcal{O}\left(\mathbb{A}^{3}\right)$ is a $G$ stable subspace. This implies that the linear projection $p: \mathbb{A}^{3} \rightarrow \mathbb{C}^{2},(x, y, z) \mapsto$ $(x, z)$ is $G$-equivariant. Now [KK96, Prop. 1] implies that the action of $G$ on $\mathbb{A}^{3}$ is linearizable.

\section{Fibrations and fiber bundles}

We start with the following definitions.

Definition 5.1. Let $X, Y, F$ be varieties. A morphism $\varphi: X \rightarrow Y$ is called fibration with fiber $F$ if $\varphi$ is flat and all fibers of $\varphi$ are reduced and isomorphic to $F$. If, in addition, $\varphi$ is an affine morphism, hence $F$ is affine, then we say that $\varphi$ is an affine fibration with fiber $F$.

A morphism $\varphi: X \rightarrow Y$ is called a fiber bundle with fiber $F$ if $\varphi$ is locally trivial in the étale topology with fiber $F$, i.e., for every $y \in Y$ there is an étale morphism $\mu: U \rightarrow Y$ such that $y \in \mu(U)$ and the $U \times \times_{Y} X \stackrel{\sim}{\rightarrow} U \times F$ over $U$.

The following problem goes back to a paper of Dolgachev-Weisfeiler [VD74].

Problem. Is it true that every (affine) fibration with fiber $\mathbb{A}^{n}$ is a fiber bundle?

After several attempts the case of $\mathbb{A}^{1}$-fibrations was solved in [KW85]. For $\mathbb{A}^{2}$ bundles there is a positive answer in case the base $Y$ is a smooth curve; see [KZ01]. We will give a short unified proof for both results, partially based on our Generic Isotriviality Theorem in section 2. Recall that every $\mathbb{A}^{1}$-bundle is locally trivial in the Zariski-topology whereas a similar statement for $\mathbb{A}^{2}$-bundles in not known (see also the following Remark 5.4).

\section{Theorem 5.2.}

(a) Let $\varphi: X \rightarrow Y$ be an affine fibration with fiber $\mathbb{A}^{1}$. If $Y$ is normal, then $\varphi$ is a fiber bundle, locally trivial in the Zariski-topology.

(b) If $\varphi: X \rightarrow Y$ is an affine fibration with fiber $\mathbb{A}^{2}$ and $Y$ a smooth curve, then $\varphi$ is a fiber bundle, locally trivial in the Zariski-topology.

Remark 5.1. The normality assumption in parts (a) and (b) is essential. Nori gave an example of an $\mathbb{A}^{1}$-bundle over the cusp $C:=\mathcal{V}\left(y^{2}-x^{3}\right) \subset \mathbb{C}^{2}$ which is not a fibration (see [KW85, Sect. 3.4]). Consider the normalization $\eta: \mathbb{A}^{1} \rightarrow C$ given by $t \mapsto\left(t^{2}, t^{3}\right)$, and define $\varphi: \mathbb{A}^{1} \rightarrow C \times \mathbb{P}^{1}$ by $t \mapsto(\eta(t), t)$. This is a closed embedding and $X:=C \times \mathbb{P}^{1} \backslash \varphi\left(\mathbb{A}^{1}\right)$ is an affine variety. If follows that the projection $p: X \rightarrow C$ is an $\mathbb{A}^{1}$-fibration, but there is no neighborhood $U$ of the singular point of $C$ such that $p^{-1}(U) \rightarrow U$ is a trivial bundle.

Remark 5.2. The main result of Kambayashi-Wright in [KW85] is a variant of our Theorem 5.2(a). In their setting $Y$ is a Noetherian scheme, $\varphi$ is faithfully flat of finite type and the fiber of every $y \in Y$ is isomorphic to $\mathbb{A}_{\kappa(y)}^{1}$. It is not difficult to see, using the generic isotriviality, that this implies our result. 


\section{GENERIC ISOTRIVIALITY AND LINEARIZATION}

Remark 5.3. The first two unknown cases are $\mathbb{A}^{3}$-fibrations over smooth curves and $\mathbb{A}^{2}$-fibrations over smooth surfaces. In his thesis Vénéreau constructed a polynomial $p(x, y, z, w)$ with the property that $p: \mathbb{C}^{4} \rightarrow \mathbb{C}$ is an $\mathbb{A}^{3}$-fibration and $(p, w): \mathbb{C}^{4} \rightarrow \mathbb{C}^{2}$ is an $\mathbb{A}^{2}$-fibration, but in both cases it is unknown if the fibration is locally trivial in a neighborhood of 0 (cf. [KZ04], [Lew13]).

Remark 5.4. At this point we should mention the following very interesting result due to Bass, Connell and Wright [BCW77]: Every $\mathbb{A}^{n}$-bundle over an affine variety which is locally trivial in the Zariski-topology has the structure of a vector bundle. As a consequence we get the following corollary.

\section{Corollary 5.3.}

(a) Let $\varphi: X \rightarrow Y$ be an affine fibration with fiber $\mathbb{A}^{1}$. If $Y$ is affine and normal, then $\varphi$ has the structure of a line bundle.

(b) If $\varphi: X \rightarrow Y$ is an affine fibration with fiber $\mathbb{A}^{2}$ and $Y$ an affine smooth curve, then $\varphi$ has the structure of a vector bundle of rank 2 .

It is clear from the definition that a fibration $\varphi: X \rightarrow Y$ with a smooth fiber $F$ is a smooth morphism (see [Har77, III.10 Def.]). In particular, $X$ is normal in case $Y$ is normal. In fact, we have an isomorphism of the completions $\widehat{\mathcal{O}}_{x} \simeq \widehat{\mathcal{O}}_{y} \llbracket t_{1}, \ldots, t_{n} \rrbracket$ where $y=\varphi(x)$ and $n=\operatorname{dim} F$.

We will also use the following well-known fact. If, for a given point $y \in Y$, there is a smooth morphism $\psi: Z \rightarrow Y$ such that $y \in \psi(Z)$ and $Z \times_{Y} X \simeq Z \times F$ over $Z$, then there is also an étale morphism $\eta: U \rightarrow Y$ with the same property.

Finally, every fiber bundle with fiber $\mathbb{A}^{1}$ is locally trivial in the Zariski-topology, because the automorphism group of $\mathbb{A}^{1}$ is a special group (see [KS92]).

The two basic results which we will need in the proof are the following. If $S$ is a ring and $n \in \mathbb{N}$, we use $S^{[n]}$ to denote the polynomial ring over $S$ in $n$ variables.

Proposition 5.4. Let $L / K$ be a field extension where char $K=0$. Let $R$ be $a$ finitely generated $K$-algebra such that $L \otimes_{K} R \simeq L^{[n]}$. If $n=1$ or $n=2$, then $R \simeq K^{[n]}$.

Using the Generic Equivalence Theorem from section 2 we get the following result.

Corollary 5.5. Every affine fibration $\varphi: X \rightarrow Y$ with fiber $\mathbb{A}^{1}$ or $\mathbb{A}^{2}$ admits an open dense set $U \subset Y$ where the fibration is a trivial bundle.

Proposition 5.6. Let $A$ be a discrete valuation ring with quotient field $Q(A)=$ $K$, maximal ideal $\mathfrak{m}$ and residue field $k=A / \mathfrak{m}$ where char $k=0$. Let $R \supset A$ be a domain, finitely generated and flat over $A$ such that $K \otimes_{A} R \simeq K^{[n]}$ and $k \otimes_{A} R=R / \mathfrak{m} R \simeq k^{[n]}$. If $n=1$ or $n=2$, then $R \simeq A^{[n]}$.

For both propositions the case $n=1$ is well known and not difficult to prove. As for the case $n=2$ the first proposition follows from the amalgamated product structure of the automorphism group of the algebra $K[x, y]$ (see [Kam75]), and the second proposition is proved in [Sat83, Thm. 1]. Another approach for the first proposition, due to the second author, is via Galois-equivariant completions (see [Rus02]). 


\section{HANSPETER KRAFT, PETER RUSSELL}

Remark 5.5. In case $n=1$ there is the following stronger version of Proposition 5.6 which does not assume that the morphism is affine; see [KW85, Prop. 1.4]. If $\varphi: X \rightarrow \operatorname{Spec} A$ is faithfully flat of finite type such that the generic fiber and the special fiber are both affine lines, then $X \stackrel{\sim}{\rightarrow}$ Spec $A[t]$.

The proof of Theorem 5.2 will be given in a series of lemmas. Let $\varphi: X \rightarrow Y$ be an affine fibration with fiber $\mathbb{A}^{n}$ where $n=1$ or $=2$. We can clearly assume that $Y$ is affine.

Lemma 5.7. There is a dense open set $U \subset Y$ such that $\varphi^{-1}(U) \rightarrow U$ is a trivial fiber bundle.

Proof. By the Generic Isotriviality Theorem in section 2 there is an étale morphism $U \rightarrow Y$ where $U$ is affine such that the bundle $U \times_{Y} X \rightarrow U$ is trivial. Therefore, $\mathbb{C}(U) \otimes_{\mathbb{C}(Y)} \mathcal{O}(X) \simeq \mathbb{C}(U)^{[n]}$, and so $\mathbb{C}(Y) \otimes_{\mathcal{O}(Y)} \mathcal{O}(X) \simeq \mathbb{C}(Y)^{[n]}$ by Proposition 5.4. Hence there is an $f \in \mathcal{O}(Y)$ such that $\mathcal{O}(X)_{f} \simeq \mathcal{O}(Y)_{f}^{[n]}$.

Lemma 5.8. Now assume that $Y$ is normal. Let $D \subset Y$ be an irreducible hypersurface such that $\mathcal{O}(Y)_{D}$ is normal. Then there is an $f \in \mathcal{O}(Y) \backslash I(D)$ such that $\varphi: X_{f} \rightarrow Y_{f}$ is a trivial bundle.

Proof. The morphism $E:=\varphi^{-1}(D) \rightarrow D$ is a fibration with fiber $\mathbb{A}^{n}$, and so $\mathbb{C}(D) \otimes_{\mathcal{O}(D)} \mathcal{O}(E) \simeq \mathbb{C}(D)^{[n]}$ by Lemma 5.7. By assumption, $A:=\mathcal{O}(Y)_{D}$ is a discrete valuation ring with quotient field $K:=\mathbb{C}(Y)$ and residue field $k:=\mathbb{C}(D)$. Moreover, $R:=\mathcal{O}(Y)_{D} \otimes_{\mathcal{O}(Y)} \mathcal{O}(X)$ is a domain, finitely generated and flat over $A$, such that $K \otimes_{A} R=\mathbb{C}(Y) \otimes_{\mathcal{O}(Y)} \mathcal{O}(X) \simeq K^{[n]}$ by Lemma 5.7, and

$$
\begin{aligned}
k \otimes_{A} R & =\mathbb{C}(D) \otimes_{\mathcal{O}(Y)_{D}} R=\mathbb{C}(D) \otimes_{\mathcal{O}(Y)} \mathcal{O}(X)=\mathbb{C}(D) \otimes_{\mathcal{O}(D)} \mathcal{O}(D) \otimes_{\mathcal{O}(Y)} \mathcal{O}(X) \\
& =\mathbb{C}(D) \otimes_{\mathcal{O}(D)} \mathcal{O}(E) \simeq \mathbb{C}(D)^{[n]}=k^{[n]}
\end{aligned}
$$

Therefore, by Proposition 5.6, we get $\mathcal{O}(Y)_{D} \otimes_{\mathcal{O}(Y)} \mathcal{O}(X) \simeq \mathcal{O}(Y)_{D}^{[n]}$, and the claim follows.

Define $Y_{\mathrm{bd}} \subset Y$ to be the union of all open subsets $U \subset Y$ such that $\varphi^{-1}(U) \rightarrow U$ is a trivial bundle.

Proof of Theorem 5.2(b). It follows from Lemma 5.8 that for a normal variety $Y$ the complement $Y \backslash Y_{\mathrm{bd}}$ has codimension at least 2. Hence, if $Y$ is a normal curve, then $Y_{\mathrm{bd}}=Y$.

Remark 5.6. We have shown more generally that for an affine $\mathbb{A}^{2}$-fibration $\varphi: X \rightarrow$ $Y$ where $Y$ is normal, the open set $Y_{\mathrm{bd}} \subset Y$ where $\varphi$ is a bundle has a complement of codimension at least 2 .

Lemma 5.9. Let $\varphi: X \rightarrow Y$ be an $\mathbb{A}^{1}$-bundle. Assume that there are two sections $\sigma, \tau: Y \rightarrow X$ such that $\sigma(y) \neq \tau(y)$ for all $y \in Y$. Then the bundle is trivial.

Proof. Given two points $a, b \in \mathbb{A}^{1}, a \neq b$, there is a uniquely defined morphism $\rho_{a, b}: \mathbb{A}^{1} \rightarrow \mathbb{C}$ such that $\rho_{a, b}(a)=0$ and $\rho_{a, b}(b)=1$, and this morphism is an isomorphism. Now define a map $\eta: X \rightarrow \mathbb{C}$ by

$$
\eta(x):=\rho_{\sigma(y), \tau(y)}(x) \quad \text { where } y:=\varphi(x) .
$$




\section{GENERIC ISOTRIVIALITY AND LINEARIZATION}

This map is well defined and induces an isomorphism on every fiber $\varphi^{-1}(y)$. We claim that $\eta: X \rightarrow \mathbb{C}$ is a morphism. This is obvious if the bundle is trivial, hence follows in general, because the $\left(\mathbb{A}^{1}-\right)$ bundle is locally trivial in Zariski-topology. Now we claim that the morphism $(\eta, \varphi): X \rightarrow \mathbb{C} \times Y$ is an isomorphism. Again, this is obvious if the bundle is trivial, and thus follows in general from the local triviality.

Proof of Theorem 5.2(a). Define $\tilde{Y}:=X \times_{Y} X$ and let $\psi: \tilde{Y} \rightarrow Y$ be the canonical morphism $\psi\left(x, x^{\prime}\right):=\varphi(x)\left(=\varphi\left(x^{\prime}\right)\right)$. By definition, $\psi$ is smooth and the pull-back fibration $\tilde{\varphi}: \tilde{X}:=\tilde{Y} \times_{Y} X \rightarrow \tilde{Y}$ has two sections $\sigma, \tau: \tilde{Y} \rightarrow \tilde{X}, \sigma\left(x, x^{\prime}\right):=\left(x, x^{\prime}, x\right)$ and $\tau\left(x, x^{\prime}\right):=\left(x, x^{\prime}, x^{\prime}\right)$. These sections are disjoint on $\tilde{Y}^{\prime}:=\tilde{Y} \backslash\{(x, x) \mid x \in X\}$, where $\psi^{\prime}: \tilde{Y}^{\prime} \rightarrow Y$ is still smooth and surjective. Now it suffices to prove that over any affine open set $U \subset \tilde{Y}^{\prime}$ the fibration $\tilde{\varphi}^{-1}(U) \rightarrow U$ is a trivial bundle.

Lemma 5.9 implies that $\varphi^{-1}\left(U_{\mathrm{bd}}\right) \simeq U_{\mathrm{bd}} \times \mathbb{A}^{1}$. Since the complement $Y \backslash Y_{\mathrm{bd}}$ has codimension at least 2 the same is true for $U \backslash U_{\mathrm{bd}}$ and for $\tilde{\varphi}^{-1}(U) \backslash \tilde{\varphi}^{-1}\left(U_{\mathrm{bd}}\right)$. But $U$ and $\tilde{\varphi}^{-1}(U)$ are normal affine varieties, and so finally we get

$$
\mathcal{O}\left(\tilde{\varphi}^{-1}(U)\right)=\mathcal{O}\left(\tilde{\varphi}^{-1}\left(U_{\mathrm{bd}}\right)\right) \simeq \mathcal{O}\left(U_{\mathrm{bd}} \times \mathbb{A}^{1}\right)=\mathcal{O}\left(U_{\mathrm{bd}}\right)[t]=\mathcal{O}(U)[t],
$$

hence $\tilde{\varphi}^{-1}(U) \simeq U \times \mathbb{A}^{1}$.

\section{References}

[BCW77] H. Bass, E. H. Connell, D. Wright, Locally polynomial algebras are symmetric algebras, Invent. Math. 38 (1976/77), no. 3, 279-299.

[BH85] H. Bass, W. Haboush, Linearizing certain reductive group actions, Trans. Amer. Math. Soc. 292 (1985), no. 2, 463-482.

[FK13] J.-P. Furter, H. Kraft, On the geometry of the automorphism group of affine $n$-space, 2013, to appear.

[FM10] J.-P. Furter, S. Maubach, A characterization of semisimple plane polynomial automorphisms, J. Pure Appl. Algebra 214 (2010), no. 5, 574-583.

[Har77] R. Hartshorne, Algebraic Geometry, Graduate Texts in Mathematics, Vol. 52, Springer-Verlag, New York, 1977. Russian transl.: P. Хартсхорн, гебрачческая геометрия, Мир, М., 1981.

[Jan03] J. C. Jantzen, Representations of Algebraic Groups, 2nd ed., Mathematical Surveys and Monographs, Vol. 107, American Mathematical Society, Providence, RI, 2003.

[Kam75] T. Kambayashi, On the absence of nontrivial separable forms of the affine plane, J. Algebra 35 (1975), 449-456.

[Kam79] T. Kambayashi, Automorphism group of a polynomial ring and algebraic group action on an affine space, J. Algebra 60 (1979), no. 2, 439-451.

[KW85] T. Kambayashi, D. Wright, Flat families of affine lines are affine-line bundles, Illinois J. Math. 29 (1985), no. 4, 672-681.

[KKMLR97] S. Kaliman, M. Koras, L. Makar-Limanov, P. Russell, $\mathbb{C}^{*}$-actions on $\mathbb{C}^{3}$ are linearizable, Electron. Res. Announc. Amer. Math. Soc. 3 (1997), 63-71 (electronic). 


\section{HANSPETER KRAFT, PETER RUSSELL}

[KZ01] S. Kaliman, M. Zaidenberg, Families of affine planes: the existence of a cylinder, Michigan Math. J. 49 (2001), no. 2, 353-367.

[KZ04] S. Kaliman, M. Zaidenberg, Vénéreau polynomials and related fiber bundles, J. Pure Appl. Algebra 192 (2004), no. 1-3, 275-286.

[Kno91] F. Knop, Nichtlinearisierbare Operationen halbeinfacher Gruppen auf affinen Räumen, Invent. Math. 105 (1991), no. 1, 217-220.

[Kra96] H. Kraft, Challenging problems on affine $n$-space, in: Séminaire Bourbaki, Vol. 1994/95, Astérisque 237 (1996), Exp. No. 802, 5, pp. 295-317.

[KK96] H. Kraft, F. Kutzschebauch, Equivariant affine line bundles and linearization, Math. Res. Lett. 3 (1996), no. 5, 619-627.

[KP85] H. Kraft, V. L. Popov, Semisimple group actions on the three-dimensional affine space are linear, Comment. Math. Helv. 60 (1985), no. 3, 466-479.

[KS92] H. Kraft, G. W Schwarz, Reductive group actions with one-dimensional quotient, Inst. Hautes Études Sci. Publ. Math. 76 (1992), 1-97.

[Kum02] S. Kumar, Kac-Moody Groups, their Flag Varieties and Representation Theory, Progress in Mathematics, Vol. 204, Birkhäuser Boston, Boston, MA, 2002.

[Lew13] D. Lewis, Vénéreau-type polynomials as potential counterexamples, J. Pure Appl. Algebra 217 (2013), no. 5, 946-957.

[Rus02] P. Russell, Some formal aspects of the theorems of Mumford-Ramanujam, in: Algebra, Arithmetic and Geometry, Part II (Mumbai, 2000), Tata Inst. Fund. Res. Stud. Math., Vol. 16, Tata Inst. Fund. Res., Bombay, 2002, pp. $557-584$.

[Sat83] A. Sathaye, Polynomial ring in two variables over a DVR: a criterion, Invent. Math. 74 (1983), no. 1, 159-168.

[Sch89] G. W. Schwarz, Exotic algebraic group actions, C. R. Acad. Sci. Paris Sér. I Math. 309 (1989), no. 2, 89-94.

[Sha66] I. R. Shafarevich, On some infinite-dimensional groups, Rend. Mat. e Appl. (5) 25 (1966), no. 1-2, 208-212.

[Sha81] И. Р. Шафаревич, О некоторых бесконечномерных группах, II, Изв. AH CCCP, Cер. мат. 45 (1981), вып. 1, 214-226. Engl. transl.: I. R. Shafarevich, On some infinite-dimensional groups, II, Math. USSR-Izv. 18 (1982), no. 1, 185-194.

[Sha95] И. Р. Шафаревич, Письмо в редакцию, Изв. АН СССР. Сер. мат. 59 (1995), вып. 3, 224. [I. R. Shafarevich, Letter to the Editors, Izv. Akad. Nauk SSSR, Ser. mat. 59 (1995), no. 3, 224 (Russian)].

[VD74] Б. Ю. Вейсфейлер, И. В. Долгачев, Унипотентные схемы групп над целостными кольцами, Изв. АН СССР, Сер. мат. 38 (1974), вып. 4, 757-799. Engl. transl.: B. J. Veŭsfeǔler, I. V. Dolgačev, Unipotent group schemes over integral rings, Math. USSR-Izv. 8 (1974), no. 4, 761-800.

[vdEVK07] A. van den Essen, Ha Huy Vui, H. Kraft, P. Russell, D. Wright, Polynomial Automorphisms and Related Topics, Lecture notes from the International School and Workshop (ICPA2006) Hanoi, October 9-20, 2006, H. Bass, Nguyen Van Chau, S. Maubach Eds., Publishing House for Science and Technology, Hanoi, 2007.

[vdK53] W. van der Kulk, On polynomial rings in two variables, Nieuw Arch. Wiskunde (3) 1 (1953), 33-41. 